\title{
Creencias sobre el aprendizaje de una lengua del futuro profesorado de inglés de Educación Primaria
}

\author{
ELVIRA BARRIOS ESPINOSA \\ Universidad de Málaga
}

Recibido: 17 de marzo de 2013 / Aceptado: 3 de junio de 2013

ISSN: $1697-7467$

\begin{abstract}
RESUMEN: Este artículo presenta un estudio destinado a investigar las creencias en torno al aprendizaje de una lengua albergadas por 307 futuros docentes de inglés de la etapa de Educación Primaria. Los datos, obtenidos mediante el Inventario de Creencias sobre el Aprendizaje de una Lengua (Beliefs About Language Learning Inventory (BALLI)) (Horwitz, 1987), fueron sometidos a procedimientos de estadística descriptiva. Los hallazgos permiten concluir que los participantes sostienen algunas creencias erróneas que el programa de formación debería abordar y confrontar con concepciones acordes con el estado actual de la cuestión en cuanto a investigación y a conocimiento profesional sobre aprendizaje de lenguas.

Palabras clave: creencias sobre el aprendizaje de lenguas, concepciones de profesorado en formación, formación del profesorado de inglés como Lengua Extranjera, Inventario de Creencias sobre el Aprendizaje de una Lengua
\end{abstract}

\section{Pre-Service Primary English Teachers' Beliefs About Language Learning}

\begin{abstract}
This article presents a study aimed at investigating the beliefs about language learning held by 307 prospective Primary English language teachers. The data, obtained by means of the Beliefs About Language Learning Inventory (BALLI) (Horwitz, 1987), were analysed through descriptive statistical procedures. The findings indicate that the participants hold some erroneous beliefs that the teaching education programme should address and confront with conceptions coherent with the current state of the art in the field of language learning research and professional knowledge.

Keywords: beliefs about language learning, pre-service teachers' conceptions, English as a Foreign Language teacher education, Beliefs About Language Learning Inventory
\end{abstract}

\section{INTRODUCCIÓN}

Un nutrido cuerpo de investigaciones en el área del pensamiento o la cognición del profesorado puso de manifiesto hace ya más de dos décadas que, al inicio de programas de preparación profesional, los futuros docentes cuentan ya con creencias profundamente arraigadas sobre la enseñanza y el aprendizaje (Kagan, 1992; Pajares, 1992); en el ámbito específico de la enseñanza de una segunda lengua (L2) o de una 
lengua extranjera (LE), numerosas investigaciones han constatado asimismo que, antes de comenzar el programa de formación reglamentario, el futuro profesorado dispone de un sistema internalizado de creencias, percepciones, concepciones y conocimientos acerca de la enseñanza y el aprendizaje, algunos de los cuales difieren -en distinta medida según los casos- de planteamientos y presupuestos de la didáctica de la LE considerados como válidos y fundamentados en la actualidad (Peacock, 2001; Barrios, 2002, 2010; Ng y Farrell, 2003; Basturkmen, Lowen y Ellis, 2004; Mattheoudakis, 2007; Basturkmen, 2012).

La relación inseparable entre creencias y práctica de aula ha sido discutida en el área de la didáctica de una L2/LE por varios autores desde hace aproximadamente 20 años (Johnson, 1992; Woods, 1996). Actualmente, aunque se acepta en términos generales que las creencias influyen decisivamente en la práctica del aula (Borg, 2011) y en la toma de decisiones del docente (Barrios, 2004; Arnett y Turnbull, 2008; Isikoglu, Basturk y Karaca, 2009), se reconoce asimismo que entre creencias y actuación docente se establece una relación interactiva en el sentido de que, aunque las creencias pueden condicionar las acciones, determinadas experiencias y procesos reflexivos sobre la actuación pueden generar modificaciones en las creencias (Breen et al., 2001; Sato y Kleinsasser, 2004; Bartels, 2005; Busch, 2010; Borg, 2011). Además, la investigación en este ámbito ha concluido que las limitaciones propias del contexto obstruyen la puesta en práctica de las creencias de los docentes de una L2/LE (Borg, 2003; Liao, 2003; Basturkmen, 2012).

Conclusiones de investigaciones realizadas al respecto señalan que estas creencias se generan fundamentalmente a partir de las imágenes e ideas relativas a la enseñanza y al aprendizaje que se acumulan a consecuencia de la experiencia de años -como alumno o alumna- en el sistema educativo (Lortie, 1975; Horwitz, 1985; Borg, 2003; Arnett y Tornbull, 2008; Isukoglu Basturk y Karaca, 2009), particularmente, según sostiene Peacock (1999), las que se adquieren como resultado del paso por la educación secundaria; de ser así, se podría conjeturar que las creencias sobre el aprendizaje y la enseñanza de una LE de quienes constituyen los participantes en este estudio, futuros docentes de inglés como LE a niños y niñas, son fruto de sus experiencias en un nivel de enseñanza en el que el alumnado posee un desarrollo cognitivo, social, afectivo, etc. muy diferente al del alumnado al que la titulación les acredita para enseñar (Rixon, 1999; Moon y Nikolov, 2000; Edelenbos, Johnstone y Kubanek; 2006). Consecuentemente, es muy probable que, de no operarse cambio en sus creencias, intenten aplicar modelos didácticos inefectivos -e incluso perjudiciales- cuando les llegue el momento de ejercer la docencia.

El comienzo de un nuevo aprendizaje profesional se realiza, pues, a partir de concepciones y representaciones que el futuro docente ha construido en el transcurso de su experiencia previa en torno a la enseñanza. Estos conocimientos, organizados en esquemas, constituyen el punto de partida y la herramienta de procesamiento de la información a la que éste accede posteriormente en los cursos de formación (Pajares, 1992; Bailey et al., 1996) y su importancia reside, por consecuencia, en su papel mediador en la interpretación que hacen los docentes en formación de la información que reciben sobre 
enseñanza y aprendizaje en cursos de capacitación, y en la traslación de esa información a la práctica del aula (Clark y Peterson, 1986; Kagan, 1992; Pajares, 1992).

Dado este hecho, se ha apuntado con frecuencia que un programa de formación debería, por tanto, tener como objetivo primordial el que los estudiantes establezcan lazos entre sus teorías personales y los contenidos del mismo, con el fin de iniciar el proceso de desarrollo profesional a partir de sus propias concepciones, conocimientos y percepciones personales. Como corolario de esta propuesta, resulta necesario que el formador conozca la perspectiva de la que parten los futuros profesores y profesoras de una LE, con el fin de ajustar y orientar de forma apropiada los contenidos del programa, hacerlos significativos y provocar conflictos e insatisfacciones en los docentes en formación en relación a sus concepciones, creencias y esquemas de partida (Bailey et al., 1996; MacDonald, Badger y White, 2001; Peacock, 2001; Mattheoudakis, 2007; Barrios, 2010; Busch, 2010; Wong, 2010; Özmen, 2012).

De otra parte, una de las conclusiones que a menudo se destaca en los estudios sobre las creencias de profesorado en formación -de relevancia capital para los formadores de este profesorado- es la considerable inflexibilidad de estas creencias, impermeables, en muchos casos, a las intervenciones de preparación profesional. En este sentido, según un conjunto de investigaciones, estas creencias operan a modo de tamiz selectivo que se encarga no sólo de filtrar sino también de validar estrategias que el futuro docente experimenta y contenidos a los que se le expone (Zeichner, Tabachnik y Densmore, 1987; Calderhead y Robson, 1990). A este respecto, Kagan (1992: 154), en una exhaustiva e influyente revisión de estudios sobre creencias de profesorado en formación, destaca en su conclusión:

$[\ldots]$ the personal beliefs and images that preservice candidates bring to programs of teacher education usually remain inflexible. Candidates tend to use the information provided in coursework to confirm rather than to confront and correct their pre-existing beliefs. Thus, a candidate's personal beliefs and images determine how much knowledge the candidate acquires from a preservice program and how it is interpreted.

De esta forma, el filtro que representan las creencias de los docentes en formación determina que la información que le aportan los cursos de formación e incluso la observación de prácticas de enseñanza sea digerida y absorbida por su propia concepción pedagógica, convirtiendo esta información en un material potencialmente contraproducente y "antieducativo" (Kagan, 1992). En este sentido, se ha argumentado -con apoyos en conclusiones investigaciones- que las ideas previas de los docentes en formación que no son abordadas pueden constituir obstáculos a la preparación profesional puesto que con ello se aumenta la probabilidad de que las nuevas concepciones y perspectivas sean integradas en estructuras de pensamiento anteriores (Kagan, 1992).

Dada la importancia de las creencias en la actuación del docente en el aula y en la interpretación que hace el futuro docente de los contenidos de los programas de preparación, el presente estudio se propuso estudiar las creencias de profesorado de inglés en formación para la etapa de Educación Primaria, partiendo de la convicción de que conocer esas ideas previas es un requisito indispensable para explorarlas y desafiar concepciones erróneas; como se ha comentado arriba, es más que probable que las creencias perjudiciales e incompatibles con tendencias que se consideran en la actualidad válidas y coherentes con el estado de la 
cuestión en el área de la didáctica de una LE incidan negativamente en la enseñanza que los docentes en formación desarrollen una vez que se conviertan en docentes en ejercicio, y, consecuentemente, en el aprendizaje de su alumnado. Es preciso, pues, contar con información sobre las creencias previas de los estudiantes para profesores con objeto de confrontarlas con alternativas profesionalmente fundamentadas.

Finalmente, como explica Barrios $(2002,2005)$, una de las conclusiones que se deriva de la revisión de estudios publicados en editoriales y revistas de prestigio sobre aspectos de la cognición del docente de L2/LE es que el ámbito geográfico y cultural del que son originarios y en el que desarrollan su labor docente la inmensa mayoría de los informantes de estas investigaciones se caracteriza por condiciones y variables muy diferentes a las de nuestro contexto. Así, se constata que abundan estudios sobre aspectos de procesos cognitivos de docentes de inglés estadounidenses y canadienses que imparten clase de L2 a alumnado en edad adulta, y sobre docentes asiáticos en el nivel de enseñanza secundaria. Dentro del contexto europeo, los estudios publicados en libros y revistas de difusión amplia entre los profesionales de la enseñanza del inglés como LE y su investigación se refieren -en una notable proporción- a docentes nativos de nacionalidad británica.

Este panorama resalta la necesidad de realizar estudios en nuestro entorno, en los que se reconozcan las peculiaridades contextuales, formativas, socio-culturales e históricas de nuestros docentes en ejercicio y en formación. Estudios reseñables relativos a creencias, conocimientos, presupuestos y actitudes de profesores y profesoras de inglés como LE en nuestro estado son los de López-Arenas (1991), Barrios (2002, 2004, 2010), Ortega Martín (2003), Madrid y Pérez Cañado (2004) y Ortega Martín y Madrid (2009). A diferencia de nuestro estudio, estas investigaciones no emplean un instrumento de obtención de datos sobre las mismas legitimado -el Belief About Language Learning Inventory (Horwitz, 1987 ) -, utilizado ampliamente para la investigación en torno a concepciones de alumnado y profesorado sobre el aprendizaje de una LE (Diab, 2006; Mattheoudakis, 2007; Busch, 2010; Wong, 2010; Fujiwara, 2011; Mohebi y Khodaday, 2011; Altan, 2006, 2012). Esta herramienta permite contrastar los datos obtenidos y las conclusiones extraídas en nuestro estudio, realizado en un contexto de formación singular, con los datos y conclusiones de los estudios sobre creencias de estudiantes para docentes de inglés realizados hasta la fecha en otros contextos y, por consiguiente, validar el conocimiento del que la profesión dispone en torno a creencias de profesorado de idiomas en formación.

Ante esta situación, nuestro estudio se propuso como objetivo investigar creencias sobre el aprendizaje de una lengua de futuro profesorado de inglés para la etapa de Educación Primaria, con la finalidad de contar con información con la que orientar su preceptiva formación en didáctica del inglés como LE y contribuir al conocimiento profesional de concepciones de profesorado de inglés en formación. Más concretamente, la investigación se propuso responder a los siguientes dos interrogantes:

- ¿Qué creencias sobre el aprendizaje de una lengua albergan los futuros profesores de inglés a niños y niñas?

- ¿Qué similitudes y diferencias se evidencian en relación a estas creencias en comparación con las identificadas por otros estudios? 


\section{MÉtodo}

\subsection{Participantes y contexto}

Los participantes de este estudio lo conforman 307 estudiantes de la Titulación de Maestro-Especialidad de Lengua Extranjera (Inglés) en la Facultad de Ciencias de la Educación de la Universidad de Málaga matriculados en la asignatura troncal (y, consecuentemente, obligatoria) denominada 'Didáctica del Idioma Extranjero' durante los cursos académicos 2008-2009, 2009-2010, 2010-2011 y 2011-2012 (Tabla 1). De los 307 participantes, 244 (79.48\%) son mujeres y $63(20.52 \%)$ hombres. Sus edades comprenden entre los 19 у 49 años (media: 24.71; mediana: 22).

Esta asignatura, de 7.5 créditos e impartida en el 1er. cuatrimestre del 3er. y último curso de la Diplomatura, representaba la única en materia de didáctica de la LE que el alumnado podía cursar en este curso. Sus contenidos abarcaban aspectos de fundamentación (concepciones acerca de una lengua y del aprendizaje de una lengua, teorías e hipótesis de adquisición de una segunda lengua, etc.) y, fundamentalmente, de metodología de la enseñanza de una lengua; aunque incluía contenidos de naturaleza general en torno a la enseñanza y el aprendizaje de una LE, la asignatura estaba orientada fundamentalmente a capacitar al alumnado para la enseñanza del inglés como LE a niños y niñas de entre 6 y 12 años. La asignatura, en los cuatro cursos académicos en los que se recogió información, fue impartida por la autora del artículo mediante una metodología que, partiendo de una concepción del docente como práctico reflexivo, favorecía la implicación de los estudiantes en procesos metacognitivos relacionados con la toma de conciencia, la recapacitación, la introspección, el análisis y la evaluación de los fenómenos educativos. De contenido relacionado con la didáctica de la LE existía en el programa de formación únicamente otra asignatura, 'Didáctica de la comunicación oral', de carácter optativo, en el $2^{\circ}$ curso de la titulación.

Tabla 1. Composición de la muestra por curso académico

\begin{tabular}{|r|c|c|}
\hline Curso académico & $\begin{array}{c}\text { Número de partici- } \\
\text { pantes }\end{array}$ & Porcentaje \\
\hline $2008-2009$ & 82 & 26.71 \\
\hline $2009-2010$ & 82 & 26.71 \\
\hline $2010-2011$ & 79 & 25.73 \\
\hline $2011-2012$ & 64 & 29.85 \\
\hline Total & 307 & 100 \\
\hline
\end{tabular}




\subsection{Instrumento}

El instrumento empleado en esta investigación es el Beliefs About Language Learning Inventory (BALLI) (Horwitz, 1987), con ligeras variantes para actualizarlo y acomodarlo a la situación de inglés como LE en nuestro estado y dentro del contexto europeo, siguiendo la recomendación de Horwitz (1999), de que, si se trata de estudiar las creencias de un grupo cultural determinado, se considere adaptar las creencias a ese grupo e incluso añadir nuevas. Así, mientras el cuestionario original consta de 34 afirmaciones -cada una de las cuales es considerada una entidad independiente-, el utilizado en nuestro estudio las amplió a 39, representativas de creencias en torno a las áreas siguientes: a) la dificultad del aprendizaje de una lengua (6 ítems); b) la naturaleza del aprendizaje de lenguas (6 ítems); c) la aptitud para el aprendizaje de una lengua extranjera (9 ítems); d) las estrategias de aprendizaje y de comunicación ( 9 ítems); y e) motivaciones y expectativas ( 9 ítems). El participante manifiesta su grado de acuerdo con la creencia expresada en cada ítem a través de una escala de tipo Likert de 5 puntos (donde $1=$ Muy de acuerdo y $5=$ Muy en desacuerdo).

Las razones siguientes motivaron la elección de esta herramienta: representa el cuestionario de mayor prestigio y más frecuentemente usado para la investigación de creencias sobre el aprendizaje de un idioma y ha sido utilizado ampliamente para explorar las creencias de discentes y docentes, tanto en ejercicio como en formación (Peacock, 2001; Barcelos, 2003; Tercanlioglu, 2005; Mattheoudakis, 2007; Busch, 2010; Wong, 2010; Altan, 2012), lo que, consecuentemente, permitía establecer comparaciones con resultados obtenidos por otras investigaciones en contextos diferentes al nuestro en torno a creencias de futuros docentes de LE.

\subsection{Obtención y análisis de datos}

El cuestionario BALLI, traducido al español para facilitar una comprensión más uniforme, se alojó en la plataforma educativa de la Universidad de Málaga, y se cumplimentó electrónicamente a través de Internet antes de que comenzara a impartirse la asignatura de 'Didáctica del Idioma Extranjero', en el mes de septiembre del 1er. cuatrimestre del 3er. y último curso de la Diplomatura. Su realización fue voluntaria y se obtuvo el permiso de los participantes para usar los datos en una investigación.

Una vez recogidos los datos, se procedió a analizarlos mediante estadísticos descriptivos (se halló el porcentaje, la frecuencia, la media y la desviación típica de los datos correspondientes a cada uno de los ítems); para el procesamiento de los datos se utilizó el programa de análisis estadístico SPSS (v.19).

\section{Hallazgos Y discusión}

A continuación se exponen los hallazgos del estudio, clasificados en las secciones en las que se divide el cuestionario; la lógica limitación en la extensión del artículo ha obligado a limitar la discusión a los hallazgos de particular relevancia. 


\subsection{La dificultad del aprendizaje de una lengua extranjera}

Los ítems 3, 4, 5, 16, 28 y 39 del cuestionario empleado indagan en las perspectivas de los encuestados en torno a la dificultad que entraña el aprendizaje de una lengua extranjera (Tabla 2).

En relación al ítem 5, más del $73 \%$ de los encuestados manifiesta confianza en su capacidad para llegar a hablar bien inglés, aunque resulta preocupante, tratándose de futuros docentes especialistas de inglés, que más de un cuarto carezca de tal confianza. Como han demostrado investigaciones sobre este ámbito, las creencias de autoeficacia son predictoras de niveles de logro de competencia en una LE o en un dominio específico de la misma (p. e.j, Mahyuddin et al., 2006; Magogwe y Oliver, 2007; Mills, Pajares y Herron, 2007; Hsieh y Schallert, 2008). En cualquier caso, el porcentaje de la muestra que se muestra confiado en su capacidad para llegar a hablar bien inglés representa una tasa bastante por encima de la que evidencian los estudiantes para docentes de inglés en otros contextos ( $c f$. Peacock, 2001; Tercanlioglu, 2005).

Un grado mayor de controversia que en los ítems anteriormente discutidos se evidencia en relación al tiempo estimado para conseguir fluidez en una LE en el supuesto de que se dedicara a su aprendizaje una hora diaria; algo más del $30 \%$ considera que podría conseguirse en un periodo de 3 a 5 años, mientras que algo más del $24 \%$ alarga el periodo a entre 5 y 10 años. De media, los encuestados de nuestro estudio parecen considerar que se necesita un espacio de tiempo menos prolongado para llegar a usar una lengua extranjera con fluidez que los docentes en formación inicial del estudio de Peacock (2001), aunque no son tan optimistas como los encuestados en las investigaciones de Altan (2012), Tercanlioglu (2005) y Wong (2010). Llama la atención, no obstante, la concepción irrealista -si tenemos en cuenta su condición de aprendientes durante años y futuros enseñantes de inglés- expresada por prácticamente un $30 \%$ de los informantes de nuestro estudio, de que, en menos de dos años y con una dedicación de una hora diaria, es posible conseguir fluidez en una LE.

En cuanto a la desigual dificultad que plantean las destrezas orales receptiva y productiva, las opiniones están muy repartidas; no obstante, a tenor de los resultados, hablar resulta a los participantes del estudio más difícil que comprender -con una diferencia de algo más de 10 puntos porcentuales-, aunque resulte significativo asimismo que casi una tercera parte de la muestra no sea capaz de decidir sobre si hablar es más fácil que entender una LE. Estos datos concuerdan aproximadamente con los obtenidos por la mayor parte de los otros estudios sobre creencias de docentes de inglés en formación (Peacock, 2001; Wong, 2010; Altan, 2012). 
Tabla 2. Tabla de hallazgos en la sección de 'La dificultad del aprendizaje de una lengua extranjera'

\begin{tabular}{|c|c|c|c|c|c|c|c|}
\hline Ítems & $\begin{array}{c}1: \\
\text { MDA }\end{array}$ & $\begin{array}{l}2: \\
\text { DA }\end{array}$ & $\begin{array}{l}3: \\
\mathrm{N}\end{array}$ & $\begin{array}{l}4: \\
\mathrm{ED}\end{array}$ & $\begin{array}{c}5: \\
\text { MED }\end{array}$ & DT & Med. \\
\hline $\begin{array}{l}\text { 3. Algunas lenguas son más fáci- } \\
\text { les de aprender que otras. }\end{array}$ & $\begin{array}{l}38.11 \\
(117) \\
\end{array}$ & $\begin{array}{l}44.63 \\
(137)\end{array}$ & $\begin{array}{c}12.70 \\
(39)\end{array}$ & $\begin{array}{l}3.26 \\
(10)\end{array}$ & $\begin{array}{l}1.30 \\
(4)\end{array}$ & .858 & 1.85 \\
\hline $\begin{array}{l}\text { 4. El inglés es una lengua extran- } \\
\text { jera: } 1 \text { : muy difícil; } 2 \text { : difícil; } 3 \text { : } \\
\text { de dificultad media; } 4 \text { : fácil; } 5 \text { : } \\
\text { muy fácil. }\end{array}$ & $\begin{array}{l}1.95 \\
(6)\end{array}$ & $\begin{array}{c}16.61 \\
(51)\end{array}$ & $\begin{array}{l}65.80 \\
(202)\end{array}$ & $\begin{array}{c}14.98 \\
(46)\end{array}$ & $\begin{array}{c}0.65 \\
(2)\end{array}$ & .648 & 2.96 \\
\hline $\begin{array}{l}\text { 5. Creo que acabaré aprendiendo } \\
\text { a hablar inglés muy bien. }\end{array}$ & $\begin{array}{c}31.27 \\
(96)\end{array}$ & $\begin{array}{l}42.35 \\
(130) \\
\end{array}$ & $\begin{array}{c}19.22 \\
(59)\end{array}$ & $\begin{array}{l}5.54 \\
(17) \\
\end{array}$ & $\begin{array}{c}1.63 \\
(5)\end{array}$ & 935 & 2.04 \\
\hline $\begin{array}{l}\text { 16. Si alguien dedicara una hora } \\
\text { al día a aprender una lengua } \\
\text { extranjera, ¿cuánto tardaría en } \\
\text { hablarla con fluidez? } 1: \text { menos } \\
\text { de } 1 \text { año; } 2 \text { : de } 1 \text { a } 2 \text { años; } 3: \text { de } \\
3 \text { a } 5 \text { años; } 4 \text { : de } 5 \text { a } 10 \text { años; } 5 \text { : } \\
\text { no se puede aprender una lengua } \\
\text { extranjera con } 1 \text { hora al día de } \\
\text { dedicación. }\end{array}$ & $\begin{array}{c}12.05 \\
(37)\end{array}$ & $\begin{array}{c}17.92 \\
(55)\end{array}$ & $\begin{array}{c}30.62 \\
(94)\end{array}$ & $\begin{array}{c}24.10 \\
(74)\end{array}$ & $\begin{array}{c}15.31 \\
(47)\end{array}$ & 1.226 & \\
\hline $\begin{array}{l}\text { 28. Es más fácil hablar que enten- } \\
\text { der una lengua extranjera. }\end{array}$ & $\begin{array}{l}8.14 \\
(25) \\
\end{array}$ & $\begin{array}{c}20.85 \\
(64)\end{array}$ & $\begin{array}{c}32.25 \\
(99)\end{array}$ & $\begin{array}{c}22.48 \\
(69)\end{array}$ & $\begin{array}{c}16.29 \\
(50)\end{array}$ & 1.176 & 3.18 \\
\hline $\begin{array}{l}\text { 39. Es más fácil leer y escribir } \\
\text { inglés que hablar y comprender el } \\
\text { inglés hablado. }\end{array}$ & $\begin{array}{c}22.80 \\
(70)\end{array}$ & $\begin{array}{l}36.16 \\
(111)\end{array}$ & $\begin{array}{c}22.48 \\
(69)\end{array}$ & $\begin{array}{c}15.64 \\
(48)\end{array}$ & $\begin{array}{l}2.93 \\
(9)\end{array}$ & 1.090 & 2.40 \\
\hline
\end{tabular}

Nota: MD: Muy de acuerdo; D: De acuerdo; N: Ni de acuerdo ni en desacuerdo; D: En desacuerdo; MD: Muy en desacuerdo; DT: Desviación típica; Med.: Media

\subsection{La naturaleza del aprendizaje de lenguas}

Los ítems $8,12,18,24,30$ y 31 del cuestionario expresan creencias en torno a un abanico de aspectos relacionados con la naturaleza del aprendizaje de lenguas (Tabla 3).

Alrededor de un $43 \%$ de los participantes se muestra convencido de la necesidad de conocer la cultura de los países de habla inglesa, aunque más de una tercera parte no se manifiesta ni de acuerdo ni en desacuerdo, lo cual supone un grado de acuerdo medio sobre esta cuestión inferior a los obtenidos por Altan (2012), Wong (2010) y Peackock (2012); la conciencia entre los encuestados de que el inglés se ha convertido en una lingua franca de interacción y de transmisión de información podría explicar este resultado.

No obstante ese reconocimiento, algo más de un $87 \%$ de los informantes admite que el contexto óptimo para el aprendizaje de una lengua es el de un país donde se hable dicha lengua, motivados probablemente por el hecho de que el grado de exposición al inglés y de oportunidades de interacción en esta lengua son, naturalmente, más elevados. No obstante, el porcentaje de acuerdo con esta creencia es, en unos 5 puntos porcentuales, inferior al resultado obtenido por Altan (2012) y entre 3 y 10 puntos porcentuales -en función del 
año cursado por los participantes- por debajo de los obtenidos por Peacock (2001), aunque superior al del estudio de Wong (2010).

En cuanto a la valoración del léxico como el componente principal del aprendizaje de una LE (ítem 18), los resultados evidencian proporciones de participantes semejantes entre los que se manifiestan acuerdo y disconformidad -algo más del $30 \%$ en cada caso-, con un porcentaje de más del $36 \%$ de los encuestados que no se muestra ni identificado ni discrepante con el mensaje de este ítem. El respaldo a esta creencia es bastante inferior al obtenido en los estudio de Peacock (2001), Mattheoudakis (2007), Wong (2010) y Altan (2012). Por su parte, la creencia de que la gramática es la parte principal del aprendizaje de una lengua, avalada por una proporción de participantes aún menor, próxima al $32 \%$, es asimismo inferior a la proporción de quienes manifiestan albergar esta percepción en los cuatro estudios anteriormente citados. La constatación -quizás por propia experiencia- de que los conocimientos gramaticales no son suficientes para garantizar competencia comunicativa puede encontrarse en el origen de esta percepción. Mayor grado de desacuerdo aún concita la afirmación de que la parte más importante del aprendizaje del inglés es aprender a traducir del español al inglés (casi un $75 \%$ de la muestra opta por la opción en desacuerdo o muy en desacuerdo), lo cual representa unos resultados coherentes con los conseguidos en las investigaciones con profesorado de inglés en formación de Peacock (2001), Mattheoudakis (2007), Wong (2010) y Altan (2012).

Tabla 3. Tabla de hallazgos en la sección de 'La naturaleza del aprendizaje de lenguas'

\begin{tabular}{|l|c|c|c|c|c|c|c|}
\hline Ítems & $\begin{array}{c}1: \\
\text { MDA }\end{array}$ & $\begin{array}{c}2: \\
\text { DA }\end{array}$ & $\begin{array}{c}3: \\
\text { N }\end{array}$ & $\begin{array}{c}4: \\
\text { ED }\end{array}$ & $\begin{array}{c}5: \\
\text { MED }\end{array}$ & DT & Med. \\
\hline $\begin{array}{l}\text { 8. Para hablar inglés es necesario } \\
\text { conocer las culturas de países en } \\
\text { los que se habla inglés. }\end{array}$ & $\begin{array}{c}8.79 \\
(27)\end{array}$ & $\begin{array}{c}34.53 \\
(106)\end{array}$ & $\begin{array}{c}35.18 \\
(108)\end{array}$ & $\begin{array}{c}16.61 \\
(51)\end{array}$ & $\begin{array}{c}4.89 \\
(15)\end{array}$ & .998 & 2.74 \\
\hline $\begin{array}{l}\text { 12. Lo mejor es aprender inglés en } \\
\text { un país en el que se hable inglés. }\end{array}$ & $\begin{array}{c}55.05 \\
(169)\end{array}$ & $\begin{array}{c}32.25 \\
(99)\end{array}$ & $\begin{array}{c}7.17 \\
(22)\end{array}$ & $\begin{array}{c}2.93 \\
(9)\end{array}$ & $\begin{array}{c}2.61 \\
(8)\end{array}$ & .927 & 1.66 \\
\hline $\begin{array}{l}\text { 18. La parte más importante del } \\
\text { aprendizaje de una lengua extranje- } \\
\text { ra es aprender el vocabulario. }\end{array}$ & $\begin{array}{c}3.91 \\
(12)\end{array}$ & $\begin{array}{c}28.01 \\
(86)\end{array}$ & $\begin{array}{c}36.48 \\
(112)\end{array}$ & $\begin{array}{c}27.69 \\
(85)\end{array}$ & $\begin{array}{c}3.91 \\
(12)\end{array}$ & .934 & 3 \\
\hline $\begin{array}{l}\text { 24. La parte más importante del } \\
\text { aprendizaje de una lengua extranje- } \\
\text { ra es aprender la gramática. }\end{array}$ & $\begin{array}{c}0.98 \\
(3)\end{array}$ & $\begin{array}{c}11.73 \\
(36)\end{array}$ & $\begin{array}{c}29.32 \\
(90)\end{array}$ & $\begin{array}{c}42.02 \\
(129)\end{array}$ & $\begin{array}{c}15.96 \\
(49)\end{array}$ & .924 & 3.60 \\
\hline $\begin{array}{l}\text { 30. Aprender una lengua extran- } \\
\text { jera es diferente a aprender otras } \\
\text { asignaturas. }\end{array}$ & $\begin{array}{c}34.20 \\
(105)\end{array}$ & $\begin{array}{c}41.69 \\
(128)\end{array}$ & $\begin{array}{c}16.61 \\
(51)\end{array}$ & $\begin{array}{c}5.21 \\
(16)\end{array}$ & $\begin{array}{c}2.28 \\
(7)\end{array}$ & .962 & 2 \\
\hline $\begin{array}{l}\text { 31. La parte más importante del } \\
\text { aprendizaje del inglés es aprender a } \\
\text { traducir del español al inglés. }\end{array}$ & $\begin{array}{c}1.95 \\
(6)\end{array}$ & $\begin{array}{c}4.23 \\
(13)\end{array}$ & $\begin{array}{c}18.89 \\
(58)\end{array}$ & $\begin{array}{c}39.74 \\
(122)\end{array}$ & $\begin{array}{l}35.18 \\
(108)\end{array}$ & .943 & 4.02 \\
\hline
\end{tabular}

Nota: MD: Muy de acuerdo; D: De acuerdo; N: Ni de acuerdo ni en desacuerdo; D: En desacuerdo; MD: Muy en desacuerdo; DT: Desviación típica; Med.: Media 


\subsection{Aptitud para las lenguas extranjeras}

Un total de 9 ítems del cuestionario pretendía recabar las perspectivas de los encuestados en relación al papel de distintas dimensiones y representaciones de un conjunto de habilidades cognitivas que se reconocen como facilitadoras del aprendizaje de una LE -la aptitud- en el aprendizaje de una LE, (Skehan, 2002; Dörnyei, 2005; Robinson, 2005) (Tabla 4).

Una mayoría de los encuestados que supera el $92 \%$ apoya la creencia -extendida popularmente- de que los niños aprenden más fácilmente una LE que los adultos, una cifra similar a la obtenida en los estudios de Peacock (2001), Tercanlioglu (2005), Mattheoudakis (2007), Wong (2010) y Altan (2012). No obstante, y a pensar de que algunas investigaciones han demostrado que un inicio temprano en el aprendizaje incide positivamente en aspectos del desarrollo y la fluidez fonológicos (Patkowski, 1990; Donato, Antonek y Tucker, 1996), esta supuesta ventaja de los niños sobre los adultos no se ve refrendada de forma incontestable por el conjunto de los estudios sobre este asunto, con la excepción de la superior capacidad de los niños de corta edad para la adquisición de los sonidos de una lengua en contextos naturales de aprendizaje (Singleton, 1989, 1995; García Lecumberri y Gallardo, 2003).

Tabla 4. Tabla de hallazgos en la sección de 'Aptitud para las lenguas extranjeras'

\begin{tabular}{|l|c|c|c|c|c|c|c|}
\hline Ítems & $\begin{array}{c}1: \\
\text { MDA }\end{array}$ & $\begin{array}{c}2: \\
\text { DA }\end{array}$ & $\begin{array}{c}3: \\
\text { N }\end{array}$ & $\begin{array}{c}4: \\
\text { ED }\end{array}$ & $\begin{array}{c}5: \\
\text { MED }\end{array}$ & DT & Med. \\
\hline $\begin{array}{l}\text { 1. Aprender una lengua extranjera es } \\
\text { más fácil para los niños que para los } \\
\text { adultos }\end{array}$ & $\begin{array}{c}69.71 \\
(214)\end{array}$ & $\begin{array}{c}23.13 \\
(71)\end{array}$ & $\begin{array}{c}3.58 \\
(11)\end{array}$ & $\begin{array}{c}1.63 \\
(5)\end{array}$ & $\begin{array}{c}1.95 \\
(6)\end{array}$ & 0.807 & 1.43 \\
\hline $\begin{array}{l}\text { 2. Hay personas que nacen con una } \\
\text { habilidad especial para aprender } \\
\text { lenguas extranjeras. }\end{array}$ & $\begin{array}{c}32.57 \\
(100)\end{array}$ & $\begin{array}{c}48.21 \\
(148)\end{array}$ & $\begin{array}{c}13.68 \\
(42)\end{array}$ & $\begin{array}{c}4.23 \\
(13)\end{array}$ & $\begin{array}{c}1.3 \\
(4)\end{array}$ & .865 & 1.93 \\
\hline $\begin{array}{l}\text { 10. Es más fácil aprender una nueva } \\
\text { lengua extranjera si se habla ya otra. }\end{array}$ & $\begin{array}{c}21.17 \\
(65)\end{array}$ & $\begin{array}{c}35.18 \\
(108)\end{array}$ & $\begin{array}{c}28.01 \\
(86)\end{array}$ & $\begin{array}{c}12.05 \\
(37)\end{array}$ & $\begin{array}{c}3.58 \\
(11)\end{array}$ & 1.061 & 2.42 \\
\hline $\begin{array}{l}\text { 17. Tengo una aptitud especial para } \\
\text { aprender lenguas extranjeras. }\end{array}$ & $\begin{array}{c}3.91 \\
(12)\end{array}$ & $\begin{array}{c}30.29 \\
(93)\end{array}$ & $\begin{array}{c}43.97 \\
(135)\end{array}$ & $\begin{array}{c}18.89 \\
(58)\end{array}$ & $\begin{array}{c}2.93 \\
(9)\end{array}$ & .866 & 2.87 \\
\hline $\begin{array}{l}\text { 20. Las mujeres son mejores que } \\
\text { los hombres para aprender lenguas } \\
\text { extranjeras. }\end{array}$ & $\begin{array}{c}3.91 \\
(12)\end{array}$ & $\begin{array}{c}4.23 \\
(13)\end{array}$ & $\begin{array}{c}30.94 \\
(95)\end{array}$ & $\begin{array}{c}15.66 \\
(48)\end{array}$ & $\begin{array}{c}45.28 \\
(139)\end{array}$ & 1.133 & 3.94 \\
\hline $\begin{array}{l}\text { 11. Las personas que son buenas } \\
\text { en matemáticas y ciencias no son } \\
\text { buenas en lenguas extranjeras. }\end{array}$ & $\begin{array}{c}1.95 \\
(6)\end{array}$ & $\begin{array}{c}2.61 \\
(8)\end{array}$ & $\begin{array}{c}18.57 \\
(57)\end{array}$ & $\begin{array}{c}26.06 \\
(80)\end{array}$ & $\begin{array}{c}50.81 \\
(156)\end{array}$ & .965 & 4.21 \\
\hline $\begin{array}{l}\text { 33. La gente que habla más de una } \\
\text { lengua extranjera es muy inteligente. }\end{array}$ & $\begin{array}{c}6.19 \\
(19)\end{array}$ & $\begin{array}{c}23.13 \\
(71)\end{array}$ & $\begin{array}{c}41.04 \\
(126)\end{array}$ & $\begin{array}{c}22.48 \\
(69)\end{array}$ & $\begin{array}{c}7.17 \\
(22)\end{array}$ & .997 & 3.01 \\
\hline $\begin{array}{l}\text { 6. Los espanoles son buenos en } \\
\text { lenguas extranjeras. }\end{array}$ & $\begin{array}{c}3.26 \\
(10)\end{array}$ & $\begin{array}{c}12.38 \\
(38)\end{array}$ & $\begin{array}{c}57.98 \\
(178)\end{array}$ & $\begin{array}{c}23.45 \\
(72)\end{array}$ & $\begin{array}{c}2.93 \\
(9)\end{array}$ & .773 & 3.10 \\
\hline $\begin{array}{l}\text { 38. Cualquiera puede aprender a } \\
\text { hablar una lengua extranjera. }\end{array}$ & $\begin{array}{c}52.12 \\
(160)\end{array}$ & $\begin{array}{c}33.88 \\
(104)\end{array}$ & $\begin{array}{c}7.49 \\
(23)\end{array}$ & $\begin{array}{c}4.56 \\
(14)\end{array}$ & $\begin{array}{c}1.95 \\
(6)\end{array}$ & .932 & 1.70 \\
\hline
\end{tabular}

Nota: MD: Muy de acuerdo; D: De acuerdo; N: Ni de acuerdo ni en desacuerdo; D: En desacuerdo; MD: Muy en desacuerdo; DT: Desviación típica; Med.: Media 


\subsection{Estrategias de aprendizaje y comunicación}

En esta sección del cuestionario (Tabla 5), tres de sus ítems (los números 7, 9 y 23) exploran las concepciones de los encuestados en torno a la precisión en el uso de la LE. Quizás atribuible a su condición de docentes de inglés en formación sea el hecho de que una mayoría de los participantes se muestre conforme con la afirmación de que es importante hablar inglés con una pronunciación excelente, en una proporción por encima de la de la muestra de Peacock (2001) y de Mattheoudakis (2007), aunque notablemente por debajo de la de otros estudios sobre creencias de docentes en formación que usan el mismo instrumento de recogida de datos (Tercanlioglu, 2005; Wong, 2010; Altan, 2012). Aunque sería preciso determinar la interpretación que los informantes hacen del concepto de 'pronunciación excelente', es razonable conjeturar que se trata de un modelo de pronunciación próximo al estándar nativo, el cual se asume, de manera irrealista, como el modelo normativo que asegura una comunicación perfecta. Frente a esa postura, y desde una consideración del inglés como lengua internacional (Seidlhofer, 2003, 2010, 2011), se propone, sin embargo, en la actualidad, que sea la consecución de inteligibilidad fonológica -y no el modelo nativo- el principio que oriente la enseñanza de la pronunciación (Jenkins, 1998, 2000, 2002).

En otro orden de cosas, a tenor de los resultados obtenidos en el ítem 23, se puede concluir que un número elevado de los participantes -en una proporción similar a la del estudio de Wong (2010) aunque menor a la que obtienen otros estudios sobre creencias de docentes en formación (Peacock, 2001; Tercanlioglu, 2005; Altan, 2012)- o bien está conforme o bien indeciso sobre la conveniencia de corregir las intervenciones incorrectas del alumnado principiante para evitar que más adelante le resulte más difícil hablar correctamente. Esta creencia, por otra parte, podría tener consecuencias significativas en la práctica del aula como, p. ej., el descarte de actividades comunicativas en el aula en las que el docente no ejerza un control estricto sobre las producciones de los alumnos.

Por último, el ítem 15 de esta sección constata la apreciación de la estrategia de compensación que representa el hacer conjeturas en caso de una laguna léxica, en relación a la cual sorprende que más de un tercio de la muestra manifieste una postura indefinida y que, de media, reconozca la adecuación del uso de esta estrategia en un grado inferior al de otros estudios (Peacock, 2001; Tercanlioglu, 2005; Wong, 2010; Altan, 2012).

Tabla 5. Tabla de hallazgos en la sección de 'Estrategias de aprendizaje y comunicación'

\begin{tabular}{|l|c|c|c|c|c|c|c|}
\hline Ítems & $\begin{array}{c}1: \\
\text { MDA }\end{array}$ & $\begin{array}{c}2: \\
\text { DA }\end{array}$ & $\begin{array}{c}3: \\
\text { N }\end{array}$ & $\begin{array}{c}4: \\
\text { ED }\end{array}$ & $\begin{array}{c}5: \\
\text { MED }\end{array}$ & DT & Med. \\
\hline $\begin{array}{l}\text { 7. Es importante hablar inglés } \\
\text { con una pronunciación excelente. }\end{array}$ & $\begin{array}{c}16.29 \\
(50)\end{array}$ & $\begin{array}{c}42.35 \\
(130)\end{array}$ & $\begin{array}{c}24.43 \\
(75)\end{array}$ & $\begin{array}{c}14.33 \\
(44)\end{array}$ & $\begin{array}{c}2.61 \\
(8)\end{array}$ & 1.010 & 2.45 \\
\hline $\begin{array}{l}\text { 9. No se debería decir nada } \\
\text { en inglés si no se sabe decirlo } \\
\text { correctamente }\end{array}$ & $\begin{array}{c}2.28 \\
(7)\end{array}$ & $\begin{array}{c}4.89 \\
(15)\end{array}$ & $\begin{array}{c}13.36 \\
(41)\end{array}$ & $\begin{array}{c}43.65 \\
(134)\end{array}$ & $\begin{array}{c}35.83 \\
(110)\end{array}$ & .944 & 4.06 \\
\hline $\begin{array}{l}\text { 13. Disfruto practicando el inglés } \\
\text { con los nativos que me encuentro } \\
\text { o conozco. (with the Americans } \\
\text { Imeet) }\end{array}$ & $\begin{array}{c}53.42 \\
(164)\end{array}$ & $\begin{array}{c}29.97 \\
(92)\end{array}$ & $\begin{array}{c}12.05 \\
(37)\end{array}$ & $\begin{array}{c}3.26 \\
(10)\end{array}$ & $\begin{array}{c}1.30 \\
(4)\end{array}$ & .900 & 1.69 \\
\hline
\end{tabular}


Tabla 5. Tabla de hallazgos en la sección de 'Estrategias de aprendizaje y comunicación' (Cont.)

\begin{tabular}{|l|c|c|c|c|c|c|c|}
\hline $\begin{array}{l}\text { 14. Disfruto practicando el inglés } \\
\text { con personas que hablan inglés y } \\
\text { que desconocen mi lengua. }\end{array}$ & $\begin{array}{c}58.31 \\
(179)\end{array}$ & $\begin{array}{c}28.99 \\
(89)\end{array}$ & $\begin{array}{c}8.79 \\
(27)\end{array}$ & $\begin{array}{c}1.30 \\
(4)\end{array}$ & $\begin{array}{c}2.61 \\
(8)\end{array}$ & .899 & 1.61 \\
\hline $\begin{array}{l}\text { 15. Es adecuado hacer conjetu- } \\
\text { ras si no se sabe una palabra en } \\
\text { inglés. }\end{array}$ & $\begin{array}{c}20.52 \\
(63)\end{array}$ & $\begin{array}{c}33.22 \\
(102)\end{array}$ & $\begin{array}{c}36.48 \\
(112)\end{array}$ & $\begin{array}{c}7.49 \\
(23)\end{array}$ & $\begin{array}{c}2.28 \\
(7)\end{array}$ & .967 & 2.38 \\
\hline $\begin{array}{l}\text { 19. Es importante repetir y practi- } \\
\text { car mucho. }\end{array}$ & $\begin{array}{c}51.47 \\
(158)\end{array}$ & $\begin{array}{c}37.13 \\
(114)\end{array}$ & $\begin{array}{c}7.82 \\
(24)\end{array}$ & $\begin{array}{c}2.93 \\
(9)\end{array}$ & $\begin{array}{c}0.65 \\
(2)\end{array}$ & .802 & 1.64 \\
\hline $\begin{array}{l}\text { 22. Me da vergüenza hablar } \\
\text { inglés con alguien. }\end{array}$ & $\begin{array}{c}15.96 \\
(49)\end{array}$ & $\begin{array}{c}33.88 \\
(104)\end{array}$ & $\begin{array}{c}16.61 \\
(51)\end{array}$ & $\begin{array}{c}19.54 \\
(60)\end{array}$ & $\begin{array}{c}14.01 \\
(43)\end{array}$ & 1.306 & 2.82 \\
\hline $\begin{array}{l}\text { 23. Si a los alumnos principiantes } \\
\text { se les permite cometer faltas en } \\
\text { inglés, les resultará difícil hablar } \\
\text { correctamente más adelante. }\end{array}$ & $\begin{array}{c}14.01 \\
(43)\end{array}$ & $\begin{array}{c}17.59 \\
(54)\end{array}$ & $\begin{array}{c}14.33 \\
(44)\end{array}$ & $\begin{array}{c}32.57 \\
(100)\end{array}$ & $\begin{array}{c}21.50 \\
(66)\end{array}$ & 1.356 & 3.30 \\
\hline $\begin{array}{l}\text { 29. Es importante practicar con } \\
\text { audiciones (CDs, podcasts...). } \\
\text { (It is important to practice with } \\
\text { cassettes or tapes). }\end{array}$ & $\begin{array}{c}51.47 \\
(158)\end{array}$ & $\begin{array}{c}38.76 \\
(119)\end{array}$ & $\begin{array}{c}6.51 \\
(20)\end{array}$ & $\begin{array}{c}1.95 \\
(6)\end{array}$ & $\begin{array}{c}1.30 \\
(4)\end{array}$ & .800 & 1.63 \\
\hline
\end{tabular}

Nota: MD: Muy de acuerdo; D: De acuerdo; N: Ni de acuerdo ni en desacuerdo; D: En desacuerdo; MD: Muy en desacuerdo; DT: Desviación típica; Med.: Media

\subsection{Motivaciones y expectativas}

Los restantes 9 ítems del cuestionario empleado en este estudio investigan las percepciones de los participantes en lo relativo a algunos factores de tipo actitudinal (Tabla 6).

Tres ítems de esta sección pretendían establecer el grado en el que los encuestados estaban motivados a aprender inglés para llegar a conocer mejor a hablantes nativos y no nativos a través de este idioma; al menos en lo relativo a la función del inglés como vehículo de relación y de conocimiento del otro, de los resultados se desprende que existe entre los participantes una motivación significativamente mayor por aprender inglés para su uso como lingua franca entre hablantes que no comparten la misma lengua que para hacerlo con hablantes nativos. Asimismo, el porcentaje de nuestra muestra que declara desear aprender inglés para conocer mejor a los hablantes nativos es bastante inferior al de los estudios de Peacock (2001), Tercanlioglu (2005) y Wong (2010), quizás porque, en el contexto europeo, el hecho de que el inglés constituye la lengua internacional de interacción se ha convertido en los últimos años en una realidad más palpable que en otros ámbitos geográficos.

Finalmente, los datos vuelven a insistir en la perspectiva internacional y en la concepción del inglés como lingua franca que sostiene el futuro profesorado participante en el estudio cuando un porcentaje cercano al $90 \%$ afirma estar de acuerdo con la afirmación de que le gustaría (o le gusta) tener amigos que no comparten su lengua materna pero con los que se pueda comunicar a través del inglés. 
Tabla 6. Tabla de hallazgos en la sección de 'Motivaciones y expectativas'

\begin{tabular}{|l|c|c|c|c|c|c|c|}
\hline Ítems & $\begin{array}{c}1: \\
\text { MDA }\end{array}$ & $\begin{array}{c}2: \\
\mathrm{DA}\end{array}$ & $\begin{array}{c}3: \\
\mathrm{N}\end{array}$ & $\begin{array}{c}4: \\
\mathrm{ED}\end{array}$ & $\begin{array}{c}5: \\
\text { MED }\end{array}$ & DT & Med \\
\hline $\begin{array}{l}\text { 21. Los españoles consideran que } \\
\text { es importante saber hablar inglés. } \\
\text { (People in my country feel...) }\end{array}$ & $\begin{array}{c}26.06 \\
(80)\end{array}$ & $\begin{array}{c}41.37 \\
(127)\end{array}$ & $\begin{array}{c}19.87 \\
(61)\end{array}$ & $\begin{array}{c}9.45 \\
(29)\end{array}$ & $\begin{array}{c}3.26 \\
(10)\end{array}$ & 1.041 & 2.22 \\
\hline $\begin{array}{l}\text { 25. Me gusta aprender inglés } \\
\text { para poder conocer mejor a los } \\
\text { británicos. }\end{array}$ & $\begin{array}{c}8.79 \\
(27)\end{array}$ & $\begin{array}{c}26.71 \\
(82)\end{array}$ & $\begin{array}{c}33.22 \\
(102)\end{array}$ & $\begin{array}{c}19.22 \\
(59)\end{array}$ & $\begin{array}{c}12.05 \\
(37)\end{array}$ & 1.139 & 2.99 \\
\hline $\begin{array}{l}\text { 26. Me gusta aprender inglés } \\
\text { para poder conocer mejor a los } \\
\text { americanos. }\end{array}$ & $\begin{array}{c}8.14 \\
(25)\end{array}$ & $\begin{array}{c}23.13 \\
(71)\end{array}$ & $\begin{array}{c}35.83 \\
(110)\end{array}$ & $\begin{array}{c}19.87 \\
(61)\end{array}$ & $\begin{array}{c}13.03 \\
(40)\end{array}$ & 1.130 & 3.07 \\
\hline $\begin{array}{l}\text { 27. Me gusta aprender inglés para } \\
\text { conocer mejor a personas no nati- } \\
\text { vas con las que pueda comunicar- } \\
\text { me a través del inglés. }\end{array}$ & $\begin{array}{c}22.15 \\
(68)\end{array}$ & $\begin{array}{c}42.02 \\
(129)\end{array}$ & $\begin{array}{c}23.13 \\
(71)\end{array}$ & $\begin{array}{c}7.82 \\
(24)\end{array}$ & $\begin{array}{c}4.89 \\
(15)\end{array}$ & 1.054 & 2.31 \\
\hline $\begin{array}{l}\text { 32. Si aprendo inglés muy bien, } \\
\text { tendré mejores oportunidades de } \\
\text { tener un buen trabajo. }\end{array}$ & $\begin{array}{c}63.19 \\
(194)\end{array}$ & $\begin{array}{c}29.97 \\
(92)\end{array}$ & $\begin{array}{c}2.93 \\
(9)\end{array}$ & $\begin{array}{c}2.28 \\
(7)\end{array}$ & $\begin{array}{c}1.63 \\
(5)\end{array}$ & .802 & 1.49 \\
\hline $\begin{array}{l}\text { 34. Deseo aprender a hablar bien } \\
\text { inglés. }\end{array}$ & $\begin{array}{c}93.16 \\
(286)\end{array}$ & $\begin{array}{c}6.19 \\
(19)\end{array}$ & $(0)$ & $\begin{array}{c}0.33 \\
(1)\end{array}$ & $\begin{array}{c}0.33 \\
(1)\end{array}$ & .370 & 1.08 \\
\hline $\begin{array}{l}\text { 35. Me gustaría (o Me gusta) } \\
\text { tener amigos británicos. }\end{array}$ & $\begin{array}{c}64.17 \\
(197)\end{array}$ & $\begin{array}{c}22.80 \\
(70)\end{array}$ & $\begin{array}{c}9.77 \\
(30)\end{array}$ & $\begin{array}{c}0.98 \\
(3)\end{array}$ & $\begin{array}{c}2.28 \\
(7)\end{array}$ & .882 & 1.54 \\
\hline $\begin{array}{l}36 . \text { Me gustaría (o Me gusta) } \\
\text { tener amigos americanos. }\end{array}$ & $\begin{array}{c}60.26 \\
(185)\end{array}$ & $\begin{array}{c}21.82 \\
(67)\end{array}$ & $\begin{array}{c}14.01 \\
(43)\end{array}$ & $\begin{array}{c}1.30 \\
(4)\end{array}$ & $\begin{array}{c}2.61 \\
(8)\end{array}$ & .951 & 1.64 \\
\hline $\begin{array}{l}\text { 37. Me gustaría (o Me gusta) } \\
\text { tener amigos que no comparten } \\
\text { mi lengua materna y con los que } \\
\text { me pueda comunicar a través del } \\
\text { inglés. }\end{array}$ & $\begin{array}{c}65.47 \\
(201)\end{array}$ & $\begin{array}{c}24.43 \\
(75)\end{array}$ & $\begin{array}{c}6.84 \\
(21)\end{array}$ & $\begin{array}{c}(1.30 \\
(4)\end{array}$ & $\begin{array}{c}1.95 \\
(6)\end{array}$ & .838 & 1.50 \\
\hline
\end{tabular}

Nota: MD: Muy de acuerdo; D: De acuerdo; N: Ni de acuerdo ni en desacuerdo; D: En desacuerdo; MD: Muy en desacuerdo; DT: Desviación típica; Med.: Media

\subsection{Discusión general}

Los hallazgos de este estudio sugieren que el alumnado universitario que accede a la titulación que le capacita para enseñar inglés en Educación Primaria lo hace provisto de una serie de preconcepciones bien definidas acerca del aprendizaje y la enseñanza de una LE, algunas de las cuales son incompatibles con una visión comunicativa y funcional de la lengua que subyace a los diseños curriculares en nuestro estado y con el modelo de enseñanza de la misma que comporta. Es el caso, p. ej., de la importancia que los participantes otorgan a la gramática y a la consecución de una pronunciación excelente, de la necesidad que perciben de proceder a la corrección de los errores cometidos por los aprendientes principiantes 
(entre los que se encuentran los alumnos de Educación Primaria) o de la reserva expresada por una parte considerable de la muestra hacia el uso de estrategias de comunicación en caso de una laguna léxica. Dado, por una parte, que la muestra de participantes conforma un grupo bastante homogéneo en cuanto a procedencia geográfica y experiencias de aprendizaje del inglés en contextos formales de enseñanza, y, por otra, la influencia contrastada por la investigación de las vivencias previas en la conformación de estas creencias, se podría deducir que estas concepciones son en buena parte producto de este equipaje compartido de experiencias de aprendizaje. De hecho, en el estudio de Little, Singleton y Silvius (1984, en Little y Singleton, 1990), los autores llegaron a la conclusión de que "past experiences, both of education in general and of language learning in particular, played a major role in shaping attitudes to language learning" (Little y Singleton, 1990: 14).

A la luz de esos hallazgos en materia de creencias de partida, la formación inicial del profesorado de inglés debería incidir en divulgar no sólo las carencias de una concepción estructuralista de la lengua y conductista del aprendizaje, y los principios que sustentan una perspectiva comunicativa de la lengua sino, fundamentalmente, las implicaciones que conlleva la traslación de esta última concepción a la práctica del aula en términos de énfasis en que la comunicación se establezca y se mantenga frente a la primacía de la corrección de formal. Las ventajas de la adopción de criterios de inteligibilidad fonológica -en contraste con criterios basados en la similitud con el modelo nativo de pronunciación (Jenkins, 1998, 2000, 2002) -, la relevancia del uso -y, consecuentemente de la enseñanza- de estrategias de comunicación que permitan superar dificultades y déficits derivados de una competencia limitada (Færch y Kasper, 1983; Tarone, 1984; Kasper y Kellerman, 1997) y la naturaleza, la interpretación y el tratamiento del error según perspectivas actuales derivadas de la noción de lengua como comunicación y de conclusiones de investigaciones sobre el tema (Corder, 1967, 1981; Ellis, 2006, 2009, 2012; Li, 2010) entendemos que deben formar parte asimismo de los contenidos tratados en el programa de formación de futuros docentes de LE.

Otra área que los hallazgos de nuestro estudio sugiere abordar en la preparación de profesorado de LE es la relativa a la incidencia de los factores afectivos en el aprendizaje de una lengua; los datos revelan que un porcentaje relevante de los informantes carece de la confianza en que un día llegará a hablar bien inglés y que, en una mayor proporción, sienten apuro al hablar este idioma. Como se ha apuntado arriba, la investigación ha establecido de manera convincente la relación estrecha entre creencias de autoeficacia y niveles de competencia en una LE. Además, como sostienen tanto la hipótesis del output comprensible (Swain, 1985; Swain y Lapkin, 1995) como la hipótesis de la interacción (Long, 1981, 1983, 1985), es un requisito imprescindible para el aprendizaje de una lengua que el aprendiente se involucre en procesos de producción que le requieran procesamiento sintáctico y en los que se haya de esforzar por negociar el significado para comunicarse de manera efectiva. El efecto inhibidor de la producción en LE de factores tales como la timidez o la vergüenza puede representar, por consiguiente, un serio obstáculo para el aprendizaje, por lo que los programas de formación deberían abordar la relevancia de los factores afectivos en el aprendizaje de una LE, cómo acometer su tratamiento en el aula y técnicas para la superación de barreras afectivas en el uso de la LE (Arnold, 1999; Horwitz, 2000; Liu, 2006).

Mención especial merece la creencia firmemente consolidada entre el futuro profesorado de que los niños aprenden una lengua más fácilmente que los adultos; como se comentó arriba, dicha creencia se ha visto confirmada de forma indiscutible cuando el estudio se ha realizado 
en contextos naturales de aprendizaje, mientras que en contextos formales no existe evidencia definitiva a partir de la cual pueda afirmarse que -en cualquier circunstancia- cuanto antes se inicie el aprendizaje de una LE más alto será el nivel de competencia en esa lengua que alcance un alumno en el futuro; ante esta circunstancia, algunos especialistas (Djigunovich y Vilke, 2000; Johnstone, 2002; Nikolov, 2000, 2009; Edelenbos, Johnstone y Kunaneck, 2006) han concluido que un inicio temprano incide en el nivel de competencia en una LE siempre que se cumplan una serie de condiciones esenciales, tales como una intensiva interacción en el aula que ascienda a 45 minutos al día durante 5 días a la semana, una ratio reducida y profesores que posean un dominio fluido del idioma y una buena pronunciación. Se hace imprescindible, por tanto, dar a conocer estos datos a futuro profesorado de inglés a niños para concienciarlos de la necesidad de que posean un elevado nivel de competencia comunicativa -particularmente en la modalidad oral-, para que ajusten las expectativas de aprendizaje de su alumnado a las condiciones en las que se imparte la LE y para que reivindiquen en su momento a la administración condiciones de enseñanza que realmente incidan en el nivel de competencia en la LE alcanzado por el alumnado.

Junto a la identificación de contenido que deben contemplar los programas de formación del profesorado de LE, es indispensable considerar la modalidad que éstos deben adoptar para que se produzca el cambio de perspectiva que favorezca la convergencia entre las concepciones del profesorado en formación y los conocimientos de los que se nutre la didáctica de una LE. A este respecto, estudios que han evidenciado transformaciones en las concepciones que futuros docentes sostienen en el transcurso de su preparación profesional atribuyen esta evolución a los propios programas de formación, los cuales comparten estos principios: adoptan una orientación reflexiva, fomentan el que los estudiantes para docentes adquieran conciencia de sus creencias, las exploren y las confronten con planteamientos contemporáneos basados teorías e investigaciones contrastadas, y procuran que estos futuros enseñantes establezcan lazos entre experiencias propias y contenidos del programa (Cabaroglu y Roberts, 2000; MacDonald, Badger y White, 2001; Mattheoudakis, 2007; Barrios, 2010; Busch, 2010; Wong, 2010; Özmen, 2012; Altan, 2012).

Finalmente, el contraste entre los hallazgos de este estudio en torno a creencias de futuros docentes y los obtenidos por estudios en otros ámbitos diferentes mediante el mismo instrumento de recogida de datos sugiere que, mientras que en relación a algunas creencias se dan ciertas similitudes en las percepciones evidenciadas por grupos de docentes en formación de distinta procedencia, en cuanto a otras, se dan disimilitudes sobre cuya influencia o dependencia del contexto en el que surgen sería necesario investigar más detenidamente.

\section{Conclusiones}

Los hallazgos de este estudio confirman que los futuros docentes de un idioma traen consigo al programa de capacitación un bagaje de creencias sobre el aprendizaje y la enseñanza de una LE que se diferencian -en desigual medida según los casos- de los planteamientos considerados actualmente válidos por la profesión de enseñantes de una LE. Asimismo, a partir del contraste de los hallazgos de este estudio con los obtenidos en ámbitos geográficos diferentes al nuestro, se puede conjeturar que ciertas creencias que sostienen futuros docentes de inglés en torno al aprendizaje de esta lengua son sensibles -si no dependientes- 
del contexto cultural en el que surgen, aunque este extremo habría que ser confirmado por investigaciones al respecto.

Estudios como el que aquí se presenta constituyen una fuente inestimable de información con la que justificar, orientar y diseñar cursos y estrategias de formación; como ha argumentado de forma convincente la bibliografía al respecto desde hace años, es imprescindible que el programa de formación establezcan relaciones significativas entre creencias originales de los docentes en formación y las evidencias y discusiones que sustentan la visión contemporánea de la enseñanza y el aprendizaje de una LE, y que provoque en ellos insatisfacciones y conflictos con sus concepciones y esquemas de partida para ocasionar la transformación de su perspectiva hacia concepciones que el programa pretende transmitir e inculcar.

Los hallazgos de nuestro estudio han de interpretarse, no obstante, con cautela; aunque pionero en nuestro estado en la investigación de creencias sostenidas por profesorado de inglés en formación mediante un cuestionario sumamente prestigiado y ampliamente utilizado en la investigación en torno a creencias sobre el aprendizaje de una LE, el Beliefs About Language Learning Inventory (BALLI), presenta, asimismo, algunas limitaciones que es necesario señalar. Una de ellas se refiere a la naturaleza de la estrategia de obtención de datos; la utilización de un cuestionario cerrado, si bien presenta ventajas como la practicidad y la posibilidad de contrastar datos con los obtenidos por otros estudios, excluye el que los informantes aborden asuntos relacionados con sus creencias que les sean personalmente significativos e impide el poder profundizar en las respuestas elegidas por los respondientes; además, no existe la certeza de que los distintos participantes interpreten de forma homogénea el contenido de los diferentes ítems ni que lo hagan conforme a la intención pretendida por el autor del cuestionario (Erickson, 1991; Pajares, 1992; Block, 1998; Barcelos, 2003). Para contrarrestar estas carencias, se hacen necesarios estudios sobre creencias que incorporen estrategias de obtención de datos de corte cualitativo, tales como entrevistas, diarios de aprendizaje, narrativas, biografías de aprendizaje, etc. Finalmente, y en otro orden de cosas, es preciso mencionar la restricción que representa que la muestra provenga de una única institución de educación superior y la consiguiente necesidad de contar con estudios que abarquen un contexto geográfico más amplio que el nuestro.

Además, los recientes cambios en los planes de estudio para la formación del profesorado de LE en la etapa de Educación Primaria en nuestro estado han supuesto una sustancial reducción de las horas destinadas al aprendizaje de la LE; esta circunstancia podría influir en las creencias que sostiene este futuro profesorado de ahora en adelante, lo cual hace aconsejable continuar realizando estudios sobre creencias en este nuevo contexto de formación.

A pesar de estas limitaciones, entendemos que se trata de un estudio novedoso que viene a llenar un vacío de investigación sobre creencias en torno al aprendizaje de una LE de futuros docentes de inglés en un contexto determinado, que contribuye al conocimiento sobre este ámbito de la cognición del docente en formación y que aporta una valiosa información para la concepción y desarrollo de formación en didáctica de una LE.

\section{REFERENCIAS BIBLIOGRÁficas}

Altan, M.Z. (2006). "Beliefs About Language Learning of Foreign Language-Major University Students", in Australian Journal of Teacher Education, 31, 2: 45-52. 
Altan M., (2012). "Pre-Service EFL Teachers' Beliefs About Foreign Language Learning", in European Journal of Teacher Education, 35: 481-93.

Arnett, K. y Turnbull, M. (2008). "Teacher beliefs in second and foreign language teaching: a state of the art review", in H.J. Sisken (ed.), From Thought to Action: Exploring Beliefs and Outcomes in the Foreign Language Program. Boston: Thomson Heinle, Boston, 9-29.

Arnold, J. (ed.) (1999). Affect in Language Learning. Cambridge: Cambridge University Press.

Bailey, K.M., Bergthold, B., Braunstein, B., Jagodzinsky Fleischman, N., Holbrook, M.P., Tuman, J., Waissbluth, X. y Zambo, L.J. (1996). "The language learner's autobiography: Examining the 'apprenticeship of observation", in D. Freeman y J.C. Richards (eds.), Teacher Learning in Language Teaching. Cambridge: Cambridge University Press, 11-29.

Barcelos, A.M.F. (2003). "Researching Beliefs about SLA: A Critical Review", in P. Kalaja y A.M.F. Barcelos (eds.), Beliefs about SLA: New Research Approaches. Norwell, MA: Kluwer, 7-33.

Bartels, N. (ed.) (2005). Applied Linguistics and Language Teacher Education. New York: Kluwer Academic.

Barrios Espinosa, M.E. (2002): El pensamiento y la actuación de futuros maestros de inglés durante su intervención didáctica en las Prácticas de Enseñanza (CD-Rom). Granada: Editorial Universidad de Granada.

Barrios Espinosa, M.E. (2004)."El contraste entre la planificación de clases y la enseñanza interactiva en la intervención docente autónoma de futuros maestros de inglés durante las prácticas de enseñanza", en Porta Linguarum, 2: 31-55, disponible en http://dialnet.unirioja. es/descarga/articulo/1129839.pdf, consultado el 21 de febrero de 2013.

Barrios Espinosa, M.E. (2005). "La investigación en torno a la cognición del profesor de L2/ LE”, en CAUCE, 28: 33-56.

Barrios Espinosa, M.E. (2010): "La evolución de creencias sobre el aprendizaje de un idioma en profesorado de inglés en formación", en R. Caballero y M.J. Pinar (eds.), Ways and Modes of Human Communication. Cuenca: Servicio de Publicaciones de la Universidad de Castilla-La Mancha y AESLA, 257-62.

Basturkmen, H. (2012). "Review of research into the correspondence between language teachers' stated beliefs and practices", in System, 40, 2: 282-95.

Basturkmen, H., Loewen, S. y Ellis, R. (2004). "Teachers' stated belief about incidental focus on form and their classroom practices", in Applied Linguistics, 25, 2: 243-272.

Block, D. (1998). "Exploring interpretations of questionnaire items", en System, 26, 3: 403-25.

Borg, S. (2003). "Teacher cognition in grammar teaching: A literature review", in Language Awareness, 12, 2: 96-108.

Borg, S. (2011). "The impact of in-service education on language teachers' beliefs", in System, 39, 3: 370-380.

Breen, M.P., Hird, B., Milton, M., Oliver, R. y Thwaite, A. (2001). "Making sense of language teaching: Teachers' principles and classroom practices", in Applied Linguistics, 22, 4: 470-501.

Busch, D. (2010). "Pre-service teacher beliefs about language learning: the second language acquisition course as an agent for change", in Language Teaching Research, 14, 3: 318-337.

Cabaroglu, N. y Roberts, J. (2000): "Development in student teachers' pre-existing beliefs during a 1-year PGCE programme", in System, 28, 3: 387-402.

Calderhead, J. y Robson, M. (1990). "Images of Teaching: Student Teachers' Early Conceptions of Classroom Practice", in Teaching and Teacher Education, 7, 1: 1-8.

Clark, M.C. y Peterson, P. (1986). "Teachers' thought processes", in M.C. Wittrock (ed.), Handbook of Research on Teaching (3rd. ed). Nueva York: Macmillan, 255-96. 
Corder, S.P. (1967). "The significance of learners' errors", en IRAL, 5: 161-70, recogido en S.P. Corder (1981). Error Analysis and Interlanguage. Oxford: Oxford University Press, 16170 .

Corder, S.P. (1981). Error Analysis and Interlanguage. Oxford: Oxford University Press.

Diab, R. L. (2006). "University Students' Beliefs About Learning English and French in Lebanon", in System, 34, 1: 80-96

Djigunovich, J.M. y Vilke, M. (2000). "Eight years after; wishful thinking or the facts of life", in J. Moon y M. Nikolov (eds.), Research into Teaching English to Young Learners. Pecs, Hungría: University Press, 67-86.

Donato R., Antonek, J. y Tucker, G.R. (1996). "Monitoring and assessing a Japanese FLES programme: ambiance and achievement", in Language Learning, 46, 3: 497-528.

Dörnyei, Z. (2005). The Psychology of the Language Learner: Individual Differences in Second Language Acquisition. Mahwah, New Jersey: Lawrence Erlbaum Associates.

Edelenbos, P., Johnstone, R. y Kubanek, A. (2006). The main pedagogical principles underlying the teaching of languages to very young learners. Bruselas: European Commission: Education and Culture. Culture and Communication Multilingualism Policy. Disponible en http://ec.europa.eu/education/policies/lang/doc/young_en.pdf, consultado el 21 de febrero de 2013.

Ellis, R. (2006). "Current issues in the teaching of grammar: An SLA perspective", in TESOL Quarterly, 40, 1: 83-107.

Ellis, R. (2009). "Corrective feedback and teacher development", in L2 Journal, 1, 3-18. Disponible en http://www.escholarship.org/uc/item/2504d6w3, consultado el 21 de febrero de 2013.

Ellis, R. (2012). Language Teaching Research and Language Pedagogy. Malden, MA.: Wiley/ Blackwell.

Erickson, F. (1991). "Advantages and disadvantages of qualitative research design on foreign language research", in B.F. Freed (ed.), Foreign language acquisition research and the classroom. Toronto, Canada: D. C. Heath, 338-53.

Fujiwara, T. (2011). "Language Learning Beliefs of Thai EFL University Students: Dimensional Structure and Cultural Variations", in Electronic Journal of Foreign Language Teaching 8,1: 87-107.

García Lecumberri, M. L. y Gallardo, F. (2003). "English Foreign Language Sounds in School Learners of Different Ages", in M.P. García Mayo y M.L. García Lecumberri (eds.), Age And The Acquisition of English as a Foreign Language. Clevedon, Avon: Multilingual Matters, 115-35.

Horwitz, E.K. (1985). "Using Student Beliefs About Language Learning And Teaching In The Foreign Language Methods Course", in Foreign Language Annals, 18, 4: 333-40.

Horwitz, E.K. (1987). "Surveying Student Beliefs About Language Learning", in A. Wenden y J. Rubin (eds.) Learner Strategies in Language Learning. Englewood Cliffs, NJ: PrenticeHall, 119-29.

Horwitz, E.K. (1999). "Cultural And Situational Influences On Foreign Language Learners' Beliefs About Language Learning: A Review Of BALLI Studies", in System 27, 4: 557-76.

Horwitz E.K. (2000). "It Ain't Over 'til It's Over: On Foreign Language Anxiety, First Language Deficits, and the Confounding of Variables", in The Modern Language Journal, 84, 2: 256-59.

Hsieh, P.H.P. y Schallert, D.L. (2008). "Implications from Self-Efficacy and Attribution Theories for an Understanding of Undergraduates Motivation in a Foreign Language Course", in Contemporary Educational Psychology, 33, 4: 513-32. 
Isikoglu, N., Basturk, R. y Karaca, F. (2009). “Assessing in-service teachers' instructional beliefs about student-centred education: A Turkish perspective", in Teaching and Teacher Education, 25, 2: 350-56.

Jenkins, J. (1998). "Which pronunciation norms and models for English as an International Language?", in ELT Journal, 52, 2: 119-26.

Jenkins, J. (2000). The Phonology of English as an International Language. Oxford: Oxford University Press.

Jenkins, J. (2002). "A Sociolinguistically Based, Empirically Researched Pronunciation Syllabus for English as an International Language", in Applied Linguistics, 23, 1: 83-10.

Johnson, K.E. (1992): "Learning to Teach: Instructional Actions and Decisions of Preservice ESL Teachers", in TESOL Quarterly, 26, 3: 507-35.

Johnstone, R. (2002). Addressing 'The Age Factor': Some Implications for Languages Policy. Estrasburgo: Council of Europe. Disponible en http://www.coe.int/T/DG4/linguistic/Source/ JohnstoneEN.pdf, consultado el 21 de febrero de 2013.

Kagan, D. (1992). "Professional Growth Among Preservice and Beginning Teachers", in Review of Educational Research, 62, 2: 129-70.

Kasper, G. y Kellerman, E. (1997). "Introduction: Approaches to Communication Strategies", in G. Kasper y E. Kellerman (eds.), Communication Strategies: Psycholinguistic and Sociolinguistic Perspectives. Londres: Longman, 1-13.

Li, S. (2010). "The Effectiveness of Corrective Feedback In SLA: A Meta-Analysis", in Language Learning, 60, 2: 309-65.

Liao, X. (2003). Chinese secondary school EFL teachers'attitudes towards communicative language teaching and their classroom practices. Tesis doctoral. University of Auckland.

Little, D. y Singleton, D. (1990). "Cognitive Style and Learning Approach", en. R. Duda y P. Riley (eds.), Learning Styles. Nancy, France: University of Nancy, 11-19.

Little, D., Singleton, D. y Silvius, W. (1984). Learning Second Languages in Ireland: Experience, Attitudes and Needs. Dublin: Trinity College, Centre for Language and Communication Studies.

Liu, M. (2006). "Anxiety in Chinese EFL Students at Different Proficiency Levels", in System, 34, 3: 301-16.

Long, M.H. (1981). "Input, Interaction and Second-Language Acquisition”, in H. Winitz (ed.), Native Language and Foreign Language Acquisition: Vol. 379. Annals of the New York Academy of Sciences. Nueva York: New York Academy of Sciences, 259-78.

Long, M.H. (1983): "Native Speaker/Non-native Speaker Conversation and the Negotiation of Comprehensible Input", in Applied Linguistics, 4, 2: 126-41.

Long, M.H. (1985): "Input and Second Language Acquisition Theory", in S. Gass y C. Madden (eds.), Input in Second Language Acquisition. Rowley, MA: Newbury House, 377-93.

López-Arenas González, J.M. (1991): Enseñando inglés en Bachillerato. Profesores expertos y noveles. Sevilla: Grupo de Investigación Didáctica. Universidad de Sevilla.

Lortie, D. (1975): Schoolteacher: A Sociological Study. Chicago: Chicago University Press.

MacDonald, M., Badger, Y. y White, G. (2001): "Changing values: what use are theories of language learning and teaching?", in Teaching and Teacher Education, 17, 8: 949-63.

Madrid, D. y Pérez Cañado, M. L. (2004): "Teacher and Student Preferences of Native and Nonnative Foreign Language Teachers", en Porta Linguarum, 2: 125-38, disponible en http:// dialnet.unirioja.es/descarga/articulo/1129932.pdf, consultado el 21 de febrero de 2013.

Magogwe, J.M. y Oliver, R. (2007). "The Relationship between Language Learning Strategies, Proficiency, Age and Self-Efficacy Beliefs: A Study of Language Learners in Botswana", in System, 35, 3: 338-52. 
Mahyuddin, R., Elias, H., Cheong, L., Muhamad, M., Noordin, N. y Abdullah, M. (2006). "The Relationship Between Students' Self-efficacy and Their Achievement”, in Jurnal Pendidik dan Pendidikan, Jil, 21: 61-71, disponible en http://web.usm.my/education/publication/4\%20 Rahi\%20(61-71).pdf, consultado el 21 de febrero de 2013.

Mattheoudakis, M. (2007). "Tracking changes in pre-service EFL teacher beliefs in Greece: A longitudinal study", in Teaching and Teacher Education, 23, 8: 1272-88.

Mills, N., Pajares, F. y Herron, C. (2007). "Self-efficacy of College Intermediate French Students: Relation to Achievement and Motivation", in Language Learning, 57, 3: 417-42.

Ng, J. y Farrell, T.S.C. (2003). "Do teachers' beliefs of grammar teaching match their classroom practices? A Singapore case study", in D. Derling, D., Brown, AQ., Low, E.L. (eds.), English in Singapore: Research on Grammar Teaching. McGraw Hill, Singapore, 128-37.

Mohebi, S. y Khodadady, E. (2011). "Investigating University Students' Beliefs about Language Learning", in RELC Journal, 42: 3: 291-304.

Moon, J. y Nikolov, M. (eds.) (2000). Research into Teaching English to Young Learners. Pecs, Hungría: University Press.

Nikolov, M. (2000). "Issues in research into early FL programmes", in J. Moon y M. Nikolov (eds.), Research into Teaching English to Young Learners. Pecs, Hungría: University Press, 11-48.

Nikolov, M. (ed.) (2009). The Age Factor and Early Language Learning (Studies on Language Acquisition). Berlín: Mouton de Gruyter.

Ortega Martín, J.L. (2003): Percepciones de profesores y alumnos sobre la importancia de los principios y técnicas que se aplican en el aula de idioma. Tesis doctoral. Granada: Universidad de Granada.

Ortega Martín, J.L. y Madrid, D. (2009). “¿Qué técnicas de comunicación oral valoran más los profesores y alumnos en la clase de inglés?", en Porta Linguarum, 12: 183-204, disponible en http://www.ugr.es/ portalin/articulos/PL_numero12/12\%20JL\%20Ortega-D\%20Madrid. pdf, consultado el 21 de febrero de 2013.

Özmen, K.S. (2012). "Exploring Student Teachers' Beliefs about Language Learning and Teaching: A Longitudinal Study", in Current Issues in Education, 15, 1: 1-15.

Pajares, M.F. (1992). "Teachers' Beliefs and Educational Research: Cleaning up a Messy Construct", in Review of Educational Research, 62, 3: 307-32.

Patkowski, M. (1990). "Age and Accent in Second Language: A Reply to James Emil Flege", in Applied Linguistics, 11, 1: 73-89.

Peacock, M. (1999). "Beliefs About Language Learning and their Relationship to Proficiency", in International Journal of Applied Linguistics, 9, 2: 247-65.

Peacock, M. (2001). "Pre-service ESL teachers' beliefs about second language learning: a longitudinal study", in System, 29, 3: 177-95.

Rixon, S. (1999). Young Learners of English: Some Research Perspectives. Harlow: Longman.

Robinson, P. (2005). "Aptitude and second language acquisition", in Annual Review of Applied Linguistics, 25: 45-73.

Sato, K. y Kleinsasser, R.C. (2004). "Beliefs, practices, and interactions of teachers in a Japanese high school English department", in Teaching and Teacher Education, 20, 8: 797-816.

Seidlhofer, B. (2003). A Concept of International English and Related Issues: From 'Real English' to 'Realistic English'?. Estrasburgo: Council of Europe. Disponible en: http://www.coe. int/T/DG4/Linguistic/Source/SeidlhoferEN.pdf, consultado el 21 de febrero.

Seidlhofer, B. (2010). "Lingua Franca English - The European Context", in A. Kirkpatrick (ed.), The Routledge Handbook of World Englishes. Abingdon: Routledge, 355-71. 
Seidlhofer, B. (2011). Understanding English as a Lingua Franca. Oxford: Oxford University Press.

Selinker, L. (1972). "Interlanguage", in IRAL: International Review of Applied Linguistics in Language Teaching, 10, 3: 209-31.

Selinker L. (1992). Rediscovering Interlanguage. London: Longman.

Sendan, F.C. y Roberts, J. (1998). "Orhan: a case study in the development of a student teacher's personal theories", in Teachers and Teaching: Theory and Practice, 4, 2: 229-44.

Singleton, D. (1989): Language Acquisition: The Age Factor. Clevedon, UK: Multilingual Matters.

Singleton, D. (1995): "Introduction: A Critical Look at the Critical Period Hypothesis in Second Language Acquisition", in D. Singleton y Z. Lengyel (eds.), The Age Factor in Second Language Acquisition. Clevedon: Multilingual Matters, 1-29.

Skehan, P. (2002). "Theorising and updating aptitude", in P. Robinson (ed.), Cognition and second language instruction. Amsterdam: John Benjamins, 69-93.

Swain, M. (1985). "Communicative competence: some roles of comprehensible input and comprehensible output in its development”, in S. Gass y C. Madden (eds.), Input in Second Language Acquisition. Rowley, MA: Newbury House, 235-256.

Swain, M. y Lapkin, S. (1995). "Problems in Output and the Cognitive Processes They Generate: A Step Towards Second Language Learning", in Applied Linguistics, 16, 3: 371-91.

Tercanlioglu, L. (2005). "Pre-service EFL Teachers' Beliefs about Foreign Language Learning and How they Relate to Gender", in Electronic Journal of Research in Educational Psychology, 3, 1: 145-62.

Wong, M.S.L. (2010). "Beliefs About Language Learning: A Study Of Malaysian Pre-Service Teachers", in RELC Journal, 41, 123-136.

Woods, D. (1996). Teacher Cognition in Language Teaching: Beliefs, Decision-Making and Classroom practice. Cambridge: Cambridge University Press.

Zeichner, K.M., Tabachnik, B.R. y Densmore, K. (1987). "Individual, institutional, and cultural influences on the development of teachers' craft knowledge", in J. Calderhead, (ed.), Exploring Teachers' Thinking. Londres: Cassell, 21-59. 